\title{
On the Riesz-Riemann-Liouville Integral
}

By E. T. Copson:

(Received 16th July 1946. Read 1st November, 1946.)

\section{\$. Inlroduction.}

In a lecture at the Oslo Congress in 1936, Marcel Riesz ${ }^{1}$ introduced an important generalisation of the Riemann-Liouville integral of fractional order. Riesz's integral $I^{a} f$ of order a is a multiple integral in $m$ variables which converges uniformly when the real part of a exceeds $m-2$ and so represents an analytic function of the complex variable $a$. This integral is important in the theory of the generalised wave equation, for it provides a direct method of solving Cauchy's initial-value problem. The most recent developments ${ }^{2}$ show that it is likely to be also of great importance in quantum electrodynamics.

Let us denote the $m$ variables which appear in Riesz's integral by $\left(t, x_{1}, x_{2}, \ldots x_{n}\right)$, or, more briefly, by $\left(l, x_{i}\right)$, so that $m=n+1$. The variable $t$ is treated separately because it is to be the time-variable in the applications of the theory. Let us denote the "interval" in the sense of special relativity between the two point-events $P\left(T ; X_{i}\right)$ and $Q\left(t ; x_{i}\right)$ by $s$, so that

$$
s^{2}=(T-t)^{2}-\sum_{i=1}^{n}\left(X_{i}-x_{i}\right)^{2} .
$$

When $P$ is fixed and $Q$ varies, the equation $s=0$ represents the light-cone of special relativity; it is the characteristic cone of the gen eralised wave equation

$$
L(u) \equiv \frac{\partial^{2} u}{\partial t^{2}}-\sum_{i=1}^{n} \frac{\partial^{2} u}{\partial x_{i}{ }^{2}}=0 .
$$

Inside the characteristic cone, $s^{2}$ is positive, outside it, negative. The half of the cone on which $t<T$ is called the retrograde cone and will be denoted by $D(P)$.

In the problem of Cauchy for the wave equation (1.2), we have to find the solution, given the values taken by $u$ and its first partial derivatives on an $n$-dimensional manifold or hypersurface $S$. $S$ is

${ }^{1} C$ mptes rendus du congrès international des mathématiciens (Oslo, 1936). Tome 2 , pp. 44-45.

2 See a letter in Nature, 157, $734(1946)$, by T. Gustafson. 
spatially-directed in the sense of special relativity; that is, the tangentplane to $S$ at any point $R$ cuts the characteristic cone with vertex $R$ only at $R$. The surface $S$ and the retrograde cone $D(P)$ bound a hypervolume which we denote by $D(P, S)$. The Riesz integral is then defined to be

$$
I^{a} f(P)=\frac{1}{H_{m}(a)} \int_{D(P, S)} f(Q) s^{a-m} d Q
$$

where $d Q$ denotes the element of hypervolume at $Q$ and

$$
H_{m}(a)=\pi^{\frac{1}{2} m-1} 2^{a-1} \Gamma\left(\frac{1}{2} a\right) \Gamma\left(\frac{1}{2} a-\frac{1}{2} m+1\right) .
$$

The integral is then an analytic function of $\alpha$, regular when RI $\alpha>\operatorname{Max}(m-2,0)$, provided that $f$ is continuous. Its characteristic properties are expressed by the equations.

$$
I^{a} I^{\beta} f=I^{a+\beta} f, \quad L I^{a+2} f=I^{a} f,
$$

where $L$ now refers to the variables $\left(T, X_{i}\right)$.

In all applications, the fundamental problem is the analytical continuation of $I^{a} f$ into the half-plane Rl $a>0$. Riesz himself has published no solution of this problem. The cases of greatest physical importance are those for which $m=3$ or 4 and $S$ is the hyperplane $t=0$; in these cases it has been shown ${ }^{1}$ that the analytical continuation can be carried out by a simple change of variable and integration by parts. Quite recently, the general case has been discussed by Fremberg, ${ }^{2}$ who uses a rather complicated change of variable which makes his work difficult to follow. In the present paper it is shown that the methods used in the simpler cases are also applicable in the general case and lead to the desired results. It is shown incidentally that, when $S$ is the hyperplane $t=-\infty, I^{m-2} f$ is simply the retarded potential.

\section{§2. A transformation of Riesz's Integral.}

Since $I^{\alpha} f(P)$ is to be defined for all positive values of $T$, the hypersurface $S$ and the retrograde cone $D(P)$ must bound a hypervolume no matter how large $T$ is. Hence $S$ cannot be a closed surface. We write the equation of $S$ in the form $S\left(t, x_{i}\right)=0$ where the function

' Baker and Copson, The Mathematical Theor! of Huygens' Principle (Oxford, 1939), pp. 60-61. Copson, Proc. Roy. Soc. Edin. (A) 71, 260-272 (1943).

= Kungl. Fysiografiska Sälsliapets i Lund Forhandlingar, Bd, 15, Nir. 27 (1945). 
$S$ and its first partial derivatives are assumed to be continuous. Then since $S$ is spatially-directed,

$$
\left(\frac{c S}{i t}\right)^{2}-\sum_{i}\left(\frac{i S}{c x_{i}}\right)^{2}>0
$$

everywhere on $S$, a relation which implies that $S$ is not a closed surface and has no singular points. The analytical continuation with respect to $a$ is to be carried out for any fixed values of $T$ and $X_{i}$. It follows that the expression on the left-hand side of (2.1) has a positive lower bound $\delta$, depending on $T$ and $X_{i}$, on the portion $S^{\prime}$ of $S$ cut off by the retrograde cone; thus on $S^{\prime}$,

$$
\left(\frac{\partial S}{\partial t}\right)^{2}-\sum_{i}\left(\frac{\partial S}{\partial x_{i}}\right)^{2} \geqq \delta>0 .
$$

No radius vector from $P$ inside $D(P)$ can touch $S$ since $S$ is spatially-directed. Moreover, every such radius vector cuts $S$ in the same number of distinct points, since $S$ has no singular points. But $S$ and $D(P)$ bound a hypervolume; hence every radius vector from $P$ inside $D(P)$ cuts $S$ in one point. We shall denote the point where the radius vector from $P\left(T ; X_{i}\right)$ through $Q\left(l ; x_{i}\right)$ cuts $S$ by $R\left(\tau ; \xi_{i}\right)$. Similar considerations show that any line parallel to the axis of $t$ cuts $S$ in one point only, so that we may write the equation of $S$ in the form $t=S\left(x_{i}\right)$. By (2.2), the inequality

$$
\sum_{i}\left(\frac{\partial S}{\partial x_{i}}\right)^{2} \leqq 1-\delta<1
$$

then holds everywhere on $S^{\prime}$.

We now change to new variables $\left(x_{1}, x_{2}, \ldots x_{m}\right)$ defined by

$$
\begin{gathered}
x_{i}=x_{i} \quad(i=1,2,3, \ldots, n=m-1) \\
x_{m}=+\sqrt{ }\left\{(T-t)^{2}-\sum_{i}\left(x_{i}-X_{i}\right)^{2}\right\}
\end{gathered}
$$

where summation with respect to $i$ is always over the range $1,2,3, \ldots n$. This is a $(1,1)$ transformation which maps the interior of the retrograde cone on $x_{m}>0$. Since

Riesz's integral becomes

$$
\frac{\partial\left(x_{1}, x_{2}, \ldots \ldots \ldots x_{n}, t\right)}{\partial\left(x_{1}, x_{2}, \ldots \ldots x_{m-1}, x_{m}\right)}=-\frac{x_{m}}{r}
$$

$$
I^{a} f\left(T, X_{i}\right)=\frac{1}{H_{m}(a)} \int f\left(T-r, x_{i}\right) \frac{x_{m}{ }^{a-m+1}}{r} d x_{1} d x_{2} \ldots d x_{m}
$$

where integration is over the hypervolume in $x_{m}>0$ bounded by the hyperplane $x_{m}=0$ and the hypersurface $\Sigma$ whose equation is 


$$
\left\{T-S\left(x_{i}\right)\right\}^{2}=\sum_{i}\left(x_{i}-X_{i}\right)^{2}+x_{m}^{2}
$$

Evidently every radius vector from $P^{\prime}\left(X_{1}, X_{2}, \ldots X_{n}, 0\right)$, which is. the image of $P$, cuts $\Sigma$ in one point $R^{\prime}$, since this radius vector is the image of a radius vector through $P$. It is convenient to use spherical polar coordinates $\left(r, \theta, \phi_{1}, \phi_{2}, \ldots \phi_{n-1}\right)$ defined by

where

$$
x_{i}=X_{i}+l_{i} r \sin \theta, \quad x_{m}=r \cos \theta
$$

$$
\begin{aligned}
l_{n}= & \cos \phi_{1}, \\
l_{n-1}= & \sin \phi_{1} \cos \phi_{2}, \\
l_{n-2}= & \sin \phi_{1} \sin \phi_{2} \cos \phi_{3}, \\
& \ldots \ldots \ldots \ldots \ldots \ldots \sin \phi_{n-2} \cos \phi_{n-1}, \\
l_{2}= & \sin \phi_{1} \sin \phi_{2} \ldots \ldots \sin \phi_{n-2} \sin \phi_{n-1} . \\
l_{1}= & \sin \phi_{1} \sin \phi_{2} \ldots \ldots \sin
\end{aligned}
$$

If we make this change in (2.4), the angle $\theta$ varies from 0 to $\frac{1}{2} \pi$, whilst the angles $\phi$ vary so that the line whose direction-cosines in $n$-dimensional space are $\left(l_{1}, l_{2}, \ldots l_{n}\right)$ sweeps out the whole solid angle $\Omega_{n}$. Hence $\phi_{1}, \phi_{2}, \ldots \phi_{n-2}$ vary from 0 to $\pi, \phi_{n-1}$ from 0 to $2 \pi$. The coordinates of $R^{\prime}$ are then

$$
\xi_{i:}=X_{i}+l_{i} \rho \sin \theta, \quad \xi_{n}=\rho \cos \theta
$$

where $\rho$ is a continuous function of the variables $\theta$ and $\phi$. With this change of variable, we have ${ }^{1}$

$I^{a} f=\frac{1}{H_{m}(\alpha)} \int_{\Omega_{n}} \int_{0}^{1+\tau} \int_{0}^{\rho} f\left(T-r, x_{i}\right) r^{\alpha-1} \cos ^{\alpha-m+1} \theta \sin ^{m-2} \theta d r d \theta d \Omega_{n}$

where

$$
d \Omega_{n}=\sin ^{n-2} \phi_{1} \sin ^{n-3} \phi_{2} \ldots \sin \phi_{n-2} d \phi_{1} d \phi_{2} \ldots d \phi_{n-1} .
$$

The total solid angle is

$$
\Omega_{n}=\int_{\Omega_{n}} d \Omega_{n}=\frac{2 \pi ! n}{\Gamma\left(\frac{1}{2} n\right)}
$$

The analytical continuation of $I^{a} f$ depends on the formula (2.8), which shows that, so far, $I_{a}^{a} f$ is regular when $\operatorname{RI} a>\operatorname{Max}(m-2,0)$.

\section{§ 3. A lemma.}

We shall carry out the analytical continuation of $I^{a} f$ by in. tegrating $(2.8)$ by parts with respect to $\theta$. In doing so, we shall need the following lemma. ${ }^{2}$

\footnotetext{
1 Cf. Baker and Copson, loc. cit., p. 60, extuition (7.41).

2 Cf. Fremberg, loc. cit., p. 270.
} 
If the first partial derivatives of $S\left(\xi_{i}\right)$ are continuous, $\partial \rho / \partial \theta=\sigma \rho \cos \theta$

where

$$
\sigma=-\frac{\Sigma l_{i} \partial S / \partial \xi_{i}}{1+\sin \theta \Sigma l_{i} \partial S / \sigma \xi_{i}} .
$$

The function $\sigma$ is continuous on $\Sigma$.

If $\rho$ is a continuous function of $\theta$, $\phi$ satisfying an equation $\psi(\rho, \theta, \phi)=0$, where $\psi$ has continuous first partial derivatives, then $\partial \rho / \partial \theta$ exists and is given by

$$
\frac{\partial \rho}{\partial \theta}=-\frac{\partial \psi}{\partial \theta} / \frac{\partial \psi}{\partial \rho}
$$

at any point where $\partial \psi / \partial \rho$ is not zero. In the present case it follows from (2.5) that $\rho$ satisfies the equation $\psi=0$ where

and so

$$
\psi=\rho-T+S\left(\xi_{i}\right), \quad \xi_{i}=X_{i}+l_{i} \rho \sin \theta,
$$

$$
\frac{\partial \rho}{\partial \theta}=-\frac{p \rho \cos \theta}{1+p \sin \theta}
$$

where

$$
p=\Sigma l_{i} \frac{\partial S}{\partial \xi_{i}}
$$

To complete the proof of the lemma, we have to show that $1+p \sin \theta$ does not vanish on $\Sigma$. Now

$$
p^{2}=\left(\Sigma l_{i} \frac{\partial S}{\partial \xi_{i}}\right)^{2} \leqq \Sigma l_{i}^{2} \cdot \Sigma\left(\frac{\partial S}{\partial \xi_{i}}\right)^{2}=\Sigma\left(\frac{\partial S}{\partial \xi_{i}}\right)^{2} \leqq 1-\delta
$$

by (2.3). Hence

$$
1+p \sin \theta \geqq 1-|p| \geqq 1-\sqrt{ }(1-\delta)>\frac{1}{2} \delta
$$

which was to be proved.

$\S 4$. The analytical continuation of $I^{a} f$.

If $f$ and $S$ have continuous partial derivatives of order $k<\frac{1}{2}(m-1)$ and if $\operatorname{Pl} a>\operatorname{Max}(0, m-2 k-2)$, then $I^{a} f$ is equal to

$$
\begin{array}{r}
\frac{1}{K_{m}(\alpha, k)}\left[\int_{\Omega_{n}} \int_{0}^{\frac{1}{2} \pi} \int_{0}^{\rho} f_{k}\left(T-r, x_{i}\right) r^{\alpha-1} \cos ^{\alpha-m+2 k+1} \theta \sin ^{m-2 k-2} \theta d r d \theta d \Omega_{n}\right. \\
\left.\quad+\int_{\Omega_{n}} \int_{0}^{\frac{1}{2} \pi} g_{k}\left(\rho, \xi_{i}\right) \rho^{\alpha} \cos ^{\alpha-m+2 k+1} \theta \sin ^{m-2 k-1} \theta d \theta d \Omega_{n}\right]
\end{array}
$$

where $\quad K_{m}(a, k)=\pi^{\frac{1}{3} m-1} 2^{a+k-1} \Gamma\left(\frac{1}{2} a\right) \Gamma\left(\frac{1}{2} a-\frac{1}{2} m+k+1\right)$

and $f_{0}=f$

$$
f_{k+1}=(m-2 k-3) f_{k}+\Sigma\left(x_{i}-X_{i}\right) \frac{\partial f_{k}}{\partial x_{i}}
$$




$$
\begin{aligned}
& g_{0}=0 \\
& \begin{aligned}
g_{k+1}=f_{k}\left(T-\rho, \xi_{i}\right) \sigma+\sigma \rho & \sin \theta \frac{\partial g_{k}}{\partial \mu}+(1+\sigma \sin \theta) \Sigma\left(\xi_{i}-X_{i}\right) \frac{\partial ! l_{k}}{\partial \xi_{i}} \\
& +\alpha \sigma g_{k} \sin \theta+(m-2 k-2) g_{k \cdot}
\end{aligned}
\end{aligned}
$$

There are two points about the recurrence formulæ which must be emphasised. In (4.2), we regard $f_{k}$ as a function of the independent variables $x_{1}, x_{2}, \ldots x_{n}, r$ and the spherical polar coordinates are introduced only when we substitute for $f_{k}$ in (4.1). Similarly in (4.3) $g_{k}$ is a function of the independent variables $\xi_{1}, \xi_{2}, \ldots \xi_{n}, \rho$; it would be more correct but more cumbrous to write

$$
\sigma=-\underset{\rho+\Sigma\left(\xi_{i}-X_{i}\right) \partial S / \partial \xi_{i}}{\rho \Sigma l_{i} \partial S / \partial \xi_{i}} \quad \sigma \sin \theta=-\frac{\Sigma\left(\xi_{i}-X_{i}\right) \partial S / \partial \xi_{i}}{\rho+\Sigma\left(\xi_{i}-X_{i}\right) \partial S / \partial \xi_{i}} .
$$

The theorem is evidently true when $k=0$. We shall prove it in the general case by induction. The functions $f_{k}$ and $g_{k}$ are continuous if the kth partial derivatives of $f$ and $S$ are continuous; for, in the construction of $f_{k}$ and $g_{k}$ by the recurrence formulæ starting with $f_{0}$ and $g_{0}$, a discontinuity could arise only from the successive derivatives of $\sigma$ and $\sigma \sin \theta$, and these are all continuous since $1+p$ $\sin \theta>\frac{1}{2} \delta>0$.

We assume then that $I^{a} f$ is equal to the expression (1.1) that the derivatives of order $k+1$ are continuous and that $\mathrm{Rl} a>m-2 k-2>1$. The expression (4.1) consists of two terms which we treat separately. The first term is

$I_{1}=\frac{1}{K_{m}(\alpha, k)} \int_{\Omega_{n}} \int_{0}^{\frac{1}{2} \pi} F_{k}(\rho, \theta) \cos ^{\alpha-m+2 k+1} \theta \sin ^{m-2 k-2} \theta d \theta d \Omega_{n}$,

where

$$
F_{k}(\rho, \theta)=\int_{0}^{\rho} f_{k}\left(T-r, x_{i}\right) r^{a-1} d r .
$$

If we integrate by parts with respect to $\theta$, we obtain

$$
I_{1}=\frac{1}{K_{m}(\alpha, k+1)} \int_{\Omega_{n}} \int_{0}^{\frac{1}{2} \pi} \cos ^{a-m+2 k+2 \theta} \frac{d}{d \theta}\left[F_{k}(\rho, \theta) \sin ^{m-2 k-3} \theta\right] d \theta d \Omega_{n},
$$

the terms at the limits vanishing ${ }^{1}$ since $\mathrm{Rla}>m-2 k-2>1$. Moreover

$$
\frac{d}{d \theta}\left[F_{k}(\rho, \theta) \sin m-2 k-3 \theta\right]
$$

If $m=2 k+3$, there is a contribution from the lower limit $\theta=0$, and this is important in $\S 6$. 


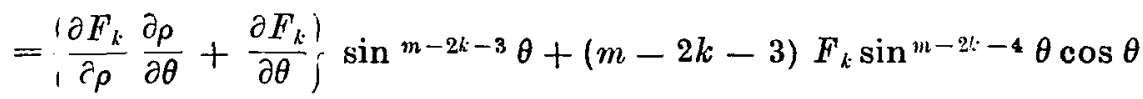

$=\left\{f_{k}\left(T-\rho, \xi_{i}\right) \sigma \rho^{a} \cos \theta+\int_{0}^{\rho} \Sigma \frac{\delta f_{k}\left(T-r, x_{i}\right)}{\partial x_{i}} \frac{\partial x_{i}}{\partial \theta} r^{a-1} d r\right\} \sin ^{m-2 k-3} \theta$

$+(n \iota-2 k-3) F_{k} \cdot \sin ^{m-2 k-4} \theta \cos \theta$

$=\left\{\bar{f}_{k} \sigma \rho^{\alpha} \cos \theta+\int_{0}^{\rho} \Sigma l_{i} r \cos \theta \frac{\partial f_{k}}{\partial x_{i}} r^{\alpha-1} d r\right\} \sin ^{m-2 k-3} \theta$

$+(m-2 k-3) F_{k} \sin ^{m-2 k-4} \theta \cos \theta$

$=\bar{f}_{k} \sigma \rho^{a} \cos \theta \sin ^{m-2 k-3} \theta+\int_{0}^{\rho} \Sigma\left(x^{i}-X_{i}\right) \frac{\partial f_{k}}{\partial x_{i}} r^{\alpha-1} d r \sin ^{m-2 k-4} \theta \cos \theta$ $+(m-2 k-3) F_{k} \cdot \sin ^{m-2 k-4} \theta \cos \theta$

$=\bar{f}_{k} \sigma \rho^{a} \cos \theta \sin ^{m-2 k-3} \theta+\int_{0}^{\rho} f_{k+1}\left(T-r, x_{i}\right) r^{a-1} d r \sin ^{m-2 k-4} \theta \cos \theta$

where $\bar{f}_{k}$ denotes $f_{k}\left(T-\rho, \xi_{i}\right)$ and where $f_{k+1}$ is defined by (4.2). We have thus proved that $I_{1}$ is equal to

$\frac{1}{K_{m}(\alpha, k+1)}\left[\iiint_{0}^{\rho} f_{k+1}\left(T-r, x_{i}\right) r^{\alpha-1} \cos ^{\alpha-m+2 k+3} \theta \sin ^{m-2 k-4} \theta d r d \theta d \Omega_{n}\right.$

$\left.+\iint \vec{f}_{k} \sigma \rho^{\alpha} \cos ^{\alpha-m+2 k+3} \theta \sin ^{m-2 k-3} \theta d \theta d \Omega_{n}\right]$.

The second term in $(4 . \bar{i})$ is

$$
\begin{aligned}
I_{2} & =\frac{1}{K_{m}(a, k)} \int_{\Omega_{n}} \int_{0}^{\frac{l}{3 \pi}} g_{k}\left(\rho, \xi_{i}\right) \rho^{\alpha} \cos ^{\alpha-m+3 k+1} \dot{\theta} \sin ^{m-2 k-1} \theta d \theta d \Omega_{n} \\
& =\frac{1}{K_{m}(a, k+1)} \int_{\Omega_{n}} \int_{0}^{\frac{\xi}{2} \pi} \cos ^{a-m+2 k+2} \theta \frac{d}{d \theta}\left[g_{k}\left(\rho, \xi_{i}\right) \rho^{\alpha} \sin ^{m-2 k-2} \theta\right] d \theta d \Omega_{n}
\end{aligned}
$$

on integrating by parts, the terms at the limits vanishing as before. Remembering that $\xi_{i}=X_{i}+l_{i} \rho \sin \theta$, we have

$\frac{d}{d \theta}\left[g_{-}\left(\rho, \xi_{i}\right) \rho^{a} \sin ^{m-2 k-2} \theta\right]$

$\left.=\frac{1}{\left(\frac{\partial g_{k}}{\partial \rho}\right.} \frac{\partial \rho}{\partial \theta}+\Sigma \frac{\partial g_{k}}{\partial \xi_{i}} \frac{\partial \xi_{i}}{\partial \theta}+\frac{a}{\rho} g_{k} \frac{\partial \rho}{\partial \theta}\right)^{2} \rho^{a} \sin ^{m-2 k-2} \theta$

$+(m-2 k-2) g_{k} \rho^{a} \sin ^{m-2 k-3} \theta \cos \theta$

$=\left\lceil\frac{\partial g_{k}}{\partial \rho} \sigma \rho \cos \theta+\Sigma l_{i} \frac{\partial g_{k}}{\partial \xi_{i}}(\rho \cos \theta+\sigma \rho \sin \theta \cos \theta)\right.$

$+g_{k} a \sigma \cos \theta_{j}^{\prime} \cdot \rho^{a} \sin { }^{m-2 k-2} \theta+(m-2 k-2) g_{k} \rho^{a} \sin ^{m-2 k-3} \theta \cos \theta$ 


$$
\begin{aligned}
=\rho^{a} \sin { }^{m-2 k-3} \theta \cos \theta\left\{\sigma \rho \frac{\partial g_{k}}{\partial \rho} \sin \theta+\right. & (1+\sigma \sin \theta) \Sigma\left(\xi_{i}-X_{i}\right) \frac{\partial g_{k}}{\partial \xi_{i}} \\
& \quad+\alpha \sigma g_{k} \sin \theta+(m-2 k-2) g_{k} \frac{1}{j} \\
=\rho^{\alpha} \sin ^{m-2 k-3} \theta \cos \theta\left\{g_{k+1}-\sigma \widehat{f_{k}}\right\} &
\end{aligned}
$$

where $g_{k+1}$ is defined by (4.3). Hence

$I_{2}=\frac{1}{K_{m}(\alpha, k+1)} \int_{\Omega} \int_{0}^{! n}\left\{g_{k+1}-\sigma \bar{f}_{k}\right\} \rho^{\alpha} \cos ^{\alpha-m+2 k+3} \theta \sin ^{m-2 k-3} \theta d \theta d \Omega_{n}$.

Adding the expressions for $I_{1}$ and $I_{2}$, we obtain

$$
\begin{array}{r}
I^{\alpha} f=\frac{1}{K_{m}(a, k+1)}\left[\int_{\Omega_{n}} \int_{0}^{\frac{1}{2} \pi} \int_{0}^{\rho} f_{k^{k}+1} r^{a-1} \cos ^{a-m+2 k+3} \theta \sin ^{m-2 k-4} \theta d r d \theta d \Omega_{n}\right. \\
\left.\quad+\int_{\Omega_{n}} \int_{0}^{\frac{1}{2} \pi} g_{k+1} \rho^{a} \cos ^{a-m+2 k+3} \theta \sin ^{m-2 k-3} \theta d \theta d \Omega_{n}\right]
\end{array}
$$

which completes the induction, apart from the consideration of the region in which (4.4) is valid. But both integrals converge uniformly with respect to $a$ when $\mathrm{Rl} a>\operatorname{Max}(0, m-2 k-4)$, and so the result is proved.

§5. The limit of $I^{a} f$ as $a \rightarrow+0$ when $m$ is even.

By $\S 4, I^{a} f$ is regular in the half-plane $\operatorname{RI} \alpha>\operatorname{Max}(0, m-2 k-2)$, provided that the derivatives of $f$ and $S$ of order $k<\frac{1}{2}(m-1)$ are continuous. It is necessary to consider separately the cases when $m$ is even or odd. If $m=2 p+1$, the largest possible value of $k$ is $p-1$; and if the derivatives of order $p-1$ are continuous, $I^{a} f$ is regular in $\mathrm{Rl} a>1$. If $m=2 p+2$, the largest possible value of $k$ is $p$; and if the derivatives of order $p$ are continuous, $I^{\alpha} f$ is regular in $\mathrm{Rl} a>0$. In the latter case, $I^{a} f$ is equal to

$$
\begin{array}{r}
\frac{a^{2}}{\pi^{p} 2^{a+p+1} \Gamma\left(\frac{1}{2} a+1\right) \Gamma\left(\frac{1}{2} a+1\right)}\left[\int_{\Omega_{n}} \int_{0}^{\frac{1}{2} \pi} \int_{0}^{\rho} f_{p} r^{a-1} \cos ^{\alpha-1} \theta d r d \theta d \Omega_{n}\right. \\
\left.+\int_{\Omega_{n}} \int_{0}^{\frac{1}{2} \pi} g_{p} \rho^{a} \cos ^{\alpha-1} \theta \sin \theta d \theta d \Omega_{n}\right] .
\end{array}
$$

By the recurrence formulæ (4.2) and (4.3), $f_{p}$ is independent of $\alpha$, whereas $g_{p}$ is a polynomial in $a$ of degree $p-1$.

The second term in square brackets in (5.1) evidently contributes nothing to the limit of $I^{a} f$ as $\alpha \rightarrow+0$. To deal with the first term, we use the following lemma of Fremberg. ${ }^{1}$

1 Fremberg, loc. cit., p. 274. I am grateful to a referee for pointing out that Fremberg's lemma, which onits reference to any "unspecified parameters", is really insufticient. The unspecified parameters are to be the angle-variables $\phi_{1}, \phi_{2}, \ldots \phi_{n-1}$. 
Let $g(x, y)$ be a function of $x$ and $y$ and certain unspecified parameters, which is absolutely integrable over $0 \leqq x \leqq a, 0 \leqq y \leqq b$ and which is continuous at $(0,0)$ uniformly with respect to the parameters.

Let $\int_{0}^{a}|g(x, y)| d x$ be bounded uniformly with respect to the parameters and with respect to $y$ in $0 \leqq y \leqq \eta \leqq b$.

Le $\int_{0}^{t} \mid g(x, y)$ 'dy be bounded uniformly with respect to the parameters and with respect to $x$ in $0 \leqq x \leqq \xi \leqq a$. Then, as $a \rightarrow+0$,

$$
a^{2} \int_{0}^{a} \int_{0}^{b} g(x, y) x^{a-1} y^{a-1} d x d y \rightarrow g(0,0),
$$

uniformly with respect to the parameters.

We have assumed throughout this work that the surface $S$ is spatially-directed. It is readily seen that this implies that $\rho$, which is a continuous function of the angle-variables $\theta$ and $\phi$, has a positive lower bound $a$. Moreover, the part of the range of integration with respect to $r$ between $a$ and $\rho$ contributes nothing to the limit of $I^{a} f$. Hence we apply the lemma to

$$
\begin{gathered}
J=\alpha^{2} \int_{0}^{\frac{1}{2} \pi} \int_{0}^{a} f_{p} r^{a-1} \cos ^{a-1} \theta d r d \theta \\
=\alpha^{2} \int_{0}^{1} \int_{0}^{a} f_{p} r^{a-1} y^{a-1} \frac{d r d y}{\sqrt{ }\left(1-y^{2}\right)}
\end{gathered}
$$

where $y=\cos \theta$. Evidently $f_{p}$, being a continuous function of $x_{i}$ and $r$, is a function of $r$ and $\theta$, and therefore of $r$ and $y$, which fulfils the conditions of the lemma; the " unspecified parameters" are the anglevariables $\phi$. Hence, as $a \rightarrow+0$,

$$
J \rightarrow\left[f_{p}\left(T-r, x_{i}\right)\right]_{\substack{r=0 \\ \theta=\frac{1}{2} \pi}}=f_{p}\left(T, X_{i}\right) .
$$

But since the limit of $J$ is independent of the variables $\phi$, we have

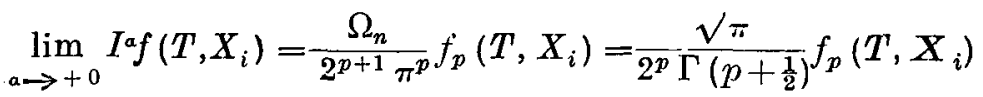

by (2.9) with $n=2 p+1$.

From (4.2), we have

$f_{k}\left(T, X_{i}\right)=(m-2 k-1) f_{k-1}\left(T, X_{i}\right)=\frac{2^{k} \Gamma\left(\frac{1}{2} m-\frac{1}{2}\right)}{\Gamma\left(\frac{1}{2} m-\frac{1}{2}-k\right)} f\left(T, X_{i}\right)$.

In the present case, $m=2 p+2$, so that

$$
f_{p}\left(T, X_{i}\right)=\frac{2^{p} \Gamma\left(p+\frac{1}{2}\right)}{\sqrt{ } \pi} f\left(T, X_{i}\right)
$$


Substituting in (5.2), we obtain the final result that

$$
\lim _{a \rightarrow+0} I^{a} f\left(T, X_{i}\right)=f\left(T, X_{i}\right)
$$

when $m=2 p+2$, provided that the partial derivatives of $f$ and $S$ of order $p$ are continuous.

$\S 6$. The limit of $I^{a} f$ as $a \rightarrow+0$ when $m$ is odd.

If $m=2 p+1$ and the derivatives of $f$ and $S^{\prime}$ of order $p-1$ are continuous, $I^{a} f$ is regular in $\mathrm{Rl} \alpha>1$ and is equal to

$$
\begin{array}{r}
\pi^{\bar{p}-\frac{1}{2^{\alpha}+p-2 \Gamma\left(\frac{1}{2} a\right)} \Gamma\left(\frac{1}{2} a-\frac{1}{2}\right)}\left[\int_{\Omega_{n}} \int_{0}^{\frac{1}{2} \pi} \int_{0}^{\rho} f_{p-1} r^{\alpha-1} \cos ^{\alpha-2} \theta \sin \theta d r d \theta d \Omega_{n}\right. \\
\left.+\int_{\Omega_{n}} \int_{0}^{\frac{1}{3 \pi}} g_{p-1} \rho^{\alpha} \cos ^{\alpha-2} \theta \sin ^{2} \theta d \theta d \Omega_{n}\right] .
\end{array}
$$

To continue the function into $\mathrm{Rl} a>0$, we have to assume that the derivatives of order $p$ are continuous and again integrate by parts with respect to $\theta$. The first term inside the square brackets is

$$
-\frac{1}{a}-1 \int_{\Omega_{n}} \int_{0}^{\frac{l}{2} \pi} F_{p-1} \frac{d \cos ^{a-1} \theta}{d \theta} d \theta d \Omega_{n},
$$

so that, when we integrate by parts, the terms at the limits do not vanish: there is a non-vanishing term arising from the lower limit $\theta=0$.

Carrying out the integration by parts, we obtain for $I^{a} f$

$$
\begin{gathered}
\frac{1}{\pi^{p-\frac{1}{2}} 2^{\alpha+p-1} \Gamma\left(\frac{1}{2} a\right) \Gamma\left(\frac{1}{2} \alpha+\frac{1}{2}\right)}\left[\int_{\Omega_{n}} \int_{0}^{\frac{1}{2} \pi} \int_{0}^{\rho} r^{\alpha} \cos ^{\alpha} \theta \Sigma l_{i} \frac{\partial f_{p-1}}{\partial x_{i}} d r d \theta d \Omega_{n}\right. \\
+\int_{\Omega_{n}}\left\{\int_{0}^{\rho} f_{p-1}\left(T-r, x_{i}\right) r^{\alpha-1} d r\right\} d \Omega_{n}+\int_{\Omega_{n}} \int_{0}^{\frac{1}{2} \pi} \sigma \rho^{\alpha} \cos ^{\alpha} \theta \bar{f}_{p-1} d \theta d \Omega_{n} \\
+\int_{\Omega_{n}} \int_{0}^{\frac{1}{2} \pi} \rho^{\alpha} \cos ^{\alpha} \theta\left\{\sigma \rho \sin \theta \frac{\partial g_{p-1}}{\partial \rho}\right. \\
\left.\left.\quad+(1+\sigma \sin \theta) \Sigma\left(\xi_{i}-X_{i}\right) \frac{\partial g_{p-1}}{\partial \xi_{i}}+\alpha \sigma g_{p-1} \sin \theta+g_{p-1}\right\} d \theta d \Omega_{n}\right]
\end{gathered}
$$

and this provides the analytical continuation into $\mathrm{RI} a>0$. When $a \rightarrow+0$, all the terms inside the square brackets, except the second, remain finite, and so contribute nothing to the limit of $I^{a} f$. As for the second term, we recall that $\rho$ is a continuous function of $\theta$ and $\phi$ with positive lower bound $a$, and the part of the range of integration 
with respect to $r$ between $a$ and $\rho$ again contributes nothing. Hence, since $x_{i}=X_{i}$ when $\theta=0$,

$$
\begin{aligned}
& \lim _{a \rightarrow+0} I^{a} f\left(T, X_{i}\right) \\
& =\lim _{a \rightarrow+0} \int_{\pi^{p-\frac{1}{2}}} \frac{a}{2^{a+p} \Gamma\left(\frac{1}{2} a+\frac{1}{2}\right) \Gamma\left(\frac{1}{2} a+1\right)} \int_{\Omega_{n}} \int_{0}^{a} f_{p-1}\left(T-r, X_{i}\right) r^{a-1} d r d \Omega_{n} \\
& =\frac{\Omega_{n}}{2^{p} \pi^{p}} \lim _{a \rightarrow+0} a \int_{0}^{a} f_{p-1}\left(T-r, X_{i}\right) r^{a-1} d r,
\end{aligned}
$$

the integrand now being independent of the angle-variables $\phi$.

To calculate this limit, we use the result that, if $g(x)$ is continuous at 0 ,

$$
\lim _{a \rightarrow+0} \alpha \int_{0}^{a} g(x) x^{a-1} d x=g(0)
$$

Hence we have

$$
\lim _{a \rightarrow+0} I^{a} f\left(T, X_{i}\right)=\frac{\Omega_{2 p}}{2^{p} \pi^{p}} f_{p-1}\left(T, X_{i}\right)=f\left(T, X_{i}\right)
$$

by (2.9) and (5.3). We have thus proved that

$$
\lim _{a \rightarrow+0} I^{a} f\left(T, X_{i}\right)=f\left(T, X_{i}\right)
$$

when $m=2 p+1$ provided that the partial derivatives of $f$ and $S$ of order $p$ are continuous. The results of $\S 5$ and $\S 6$ agree with those of Fremberg.

\section{§ 7. Retarded potentials.}

The transformation (2.4) of Riesz's integral leads to an interesting generalisation of the ordinary retarded potential. Let us suppose that $m \geqq 4$, that $f$ and $\partial f / \partial t$ are continuous and that $S$ is the hyperplane $t=-a$. Then, when $\operatorname{Rl} \alpha>m-2$,

$$
I^{a} f=\frac{1}{H_{m}(a)} \int_{V} f\left(T-r, x_{i}\right) \frac{x_{m}^{a-m+1}}{r} d x_{1} d x_{2} \ldots d x_{m},
$$

where integration is over the hypervolume $V$ bounded by $x_{m}=0$, $r=T+a$. We shall denote the boundary of $V$ by $\Sigma$ and the directioncosines of the outward normal by $\left(\lambda_{1}, \ldots \lambda_{m}\right)$.

Integrating by parts, we have

$$
\begin{aligned}
I^{a} f=\frac{1}{(a-m+2) H_{m}(a)}\{ & \int_{\Sigma} f\left(T-r, x_{i}\right) \frac{x_{m}^{a-m+2}}{r} \lambda_{m} d \Sigma \\
& \left.-\int_{V} x_{m}^{a-m+2} \frac{\partial}{\partial x_{m}}\left(\frac{f}{r}\right) d x_{1} d x_{2} \ldots \ldots d x_{m}\right\} .
\end{aligned}
$$


But since $\mathrm{Rl} a>m-2$, the portion $\Sigma_{1}$ of $\Sigma$ on which $x_{m}$ vanishes makes no contribution, and we may replace integration over $\Sigma$ by integration over $\Sigma_{2}$, the curved part of $\Sigma$. The resulting formula provides the analytical continuation into $\mathrm{RL} a>m-4$, as may be seen by introducing spherical polar coordinates.

In particular, we have

$$
\begin{aligned}
I^{m-2} f=\frac{1}{2^{m-2} \pi^{\frac{1}{2 m-1}} \Gamma\left(\frac{1}{2} m-1\right)} & {\left[\int_{-: 2}^{f\left(T-r, x_{i}\right)} \lambda_{m} d \Sigma\right.} \\
& \left.-\int_{V} \frac{\partial}{c x_{m}}\left\{\frac{f\left(T-r, x_{i}\right)}{r}\right\} d x_{1} d x_{2} \ldots d x^{m}\right] .
\end{aligned}
$$

If we integrate by parts again and remember that $\lambda_{m}=-1$ on $\Sigma_{1}$, we obtain

$$
I^{m-2} f=\frac{1}{2^{m-2} \pi^{\frac{1}{2} m-1} \Gamma\left(\frac{1}{2} m-1\right)} \int_{\mathbf{s}_{1}} \frac{f\left(T-r, x_{i}\right)}{r} d \Sigma .
$$

On $\Sigma_{1}, d \Sigma=d x_{1} d x_{2} \ldots d x_{n}$ and integration is over

$$
r^{2}=\sum_{1}^{n}\left(x_{i}-X_{i}\right)^{2} \leqq(T+a)^{2} .
$$

Lastly, if we make $a \rightarrow+\infty$, we have

$$
I^{m-2} f\left(T, X_{i}\right)=\frac{1}{2^{m-2} \pi^{\frac{1}{m-1}} \Gamma\left(\frac{1}{2} m-1\right)} \int \frac{f\left(T-r, x_{i}\right)}{r} d x_{1} d x_{2} \ldots d x_{n},
$$

where integration is over the whole $n$-dimensional space. This formula is a generalisation of the ordinary retarded potential, to which it reduces when $m=4$. For in the latter case we have

$$
I^{2} f=\frac{1}{4 \pi} \int \frac{f\left(T-r, x_{i}\right)}{r} d x_{1} d x_{2} d x_{3}
$$

which is a solution of $L u=f$, since $L I^{2} f=I^{0} f=f$.

University College (Dundee),

UNIVERSITY OF ST ANDREWS. 